\title{
Synergistic Study of Alzheimer Diseased Brain MRI with PET and SPECT Images using Shape based Registration and Fuzzy-Dempster Shafer Evidence Accumulation Model
}

\author{
Dipankar Ray \\ Department of CSE \\ Indian School of Mines \\ Dhanbad - 826 004, India.
}

\author{
D Dutta Majumder \\ Institute of Cybernetics and \\ Information Technology \\ Kolkata - 700 108, India. \\ and \\ Indian Statistical Institute \\ Kolkata - 700 105, India
}

\author{
Amit Das \\ Department of Computer \\ Technology \\ Bengal Engineering Science and \\ Technology University \\ Howrah- 7111103
}

\begin{abstract}
Synergistic studies of anatomical to functional imaging provide some additional information which is not always available in either of the two individual images. In case of Alzheimer disease, synergistic study of MR-PET or MR-SPECT brain images provides clinical information of functional behaviour of effected brain regions with the pathological status of corresponding tissues. But it requires alignment and fusion of two different types of imaging modalities. In our endeavour we have suggested a shape based generalised transformation model for the brain image registration and implemented it with radial basis function (RBF) neural network. For fusion we have suggested a two step process. Firstly, we have used fuzzy c-means to segment the candidate images and to assign basic probability assignment (bpa) to each pixel corresponding to each cluster. Then on the basis of these weightage (bpa) we have proposed Dempster Shafer evidence accumulation concept to combine each pixel to generate a fused image. From the experimental results it is evident that fused MRPET and MR-SPECT images can provide sufficient information for the synergistic studies of Alzheimer Diseased brain images.
\end{abstract}

Keywords: Multimodal Medical Image Registration, Synergistic study of AD, Medical Image Fusion.

\section{INTRODUCTION}

Analysis of brain functions of an Alzheimer Disease (AD) patient requires visualization of the effected areas as well as an estimation of the severity of the damaged brain tissues. Imaging technologies are helpful to identify $\mathrm{AD}$ plaques and tangles and are now rapidly becoming more powerful and precise. With PET/SPECT and MR images it is also possible to identify changes in brain structure or size which are indicative of AD. While with SPECTTl (Thallium-201) imagery it is possible to identify the hypometabolism of the effected areas, MRI provides the distinct atrophy of the same. Thus MR and PET/SPECT together give synergistic information about the structure and function of the brain. The soft-tissue image of MR augments the PET/SPECT-T1 by relating brain metabolism level to damaged brain tissue and improves anatomical localization of brain's functional activities $[1,2]$.

At present fusion of registered images are performed by different computational techniques [3]. Existing methods of superimposing of images often suffer from loss of information due to improper combination of pixels of images. Dempster Shafer (DS) theory of evidence accumulation and fusion is an effective tool for combining accumulative evidence or for changing prior evidence in the light of new evidence and has been successfully used in other areas of pattern recognition and classification [4,5]. In most of the cases it improves the reliability of decision process. As a registration tool, we have suggested a generalized transformation of the target objects on the basis of corresponding shapes from both the images and implemented them using RBF neural network model. In the sections 2 and 3 we have discussed about the anatomical to functional registration approach and the fusion methodology. Section 4 and 5 has covered the proposed methodology and the experimental results respectively.

\section{MULTIMODAL MEDICAL IMAGE REGISTRATION}

In order to perform MRI-PET/SPECT registrations, few specific features of each modality should be kept in mind. These include good resolution, sufficient contrast and least artifacts of the images. Moreover, field of volume (FOV) should cover the relevant parts of the body sufficiently in both the images.

MR images are usually reconstructed to $256 \times 256$ pixel matrix with pixel size $1 \mathrm{~mm}$. Depending on the organ of investigation the slice thickness varies between 1 and $10 \mathrm{~mm}$. To avoid motion artifacts few slices with higher thickness are acquired. Axial orientation is often preferred for brain studies but for specific requirement coronal and sagittal orientations are also considered.

PET images are usually captured as $128 \times 128$ matrix with pixel size 2 to $4 \mathrm{~mm}$ or higher. The slice thickness and the number of slices also vary and depend on the imaging devices and objects. The image orientation is usually restricted to transaxial slices and FOV is often limited to $16-25 \mathrm{~cm}$.

Reconstructed SPECT images typically have resolution of $64 \times 64$ or $128 \times 128$ pixels with pixel sizes ranging from $3-6 \mathrm{~mm}$. The number of projections for a brain scan is chosen to be approximately (equal to the width of the resulting images) 64 .

Due to these varying parameters in different images the resulting reconstructed mapped image suffers from poor resolution, noise and is susceptible to artifacts. The differences of resolution, orientation and FOV of PET/SPECT make their comparison and subsequent registration, a challenging task. To perform accurate 
spatial registration, it is necessary to consider following requisites for both the images:

1. knowledge of pixel size and inter-slice distance

2. primary orientation of the images

3. sufficient commonality in the parts of the body or organ covered by both the modalities

4. least artifacts due to involuntary movement (the most severe problems in registration of PET/SPECT and MRI) in both the modalities of images

Artifacts are the most common causes of problem in registration of PET/SPECT and MR images. The signatures of motion artifacts are different for PET/SPECT and MRI. In PET/SPECT patient movement between steps of image acquisition protocol may corrupt the images completely. But in case of MRI, artifacts are dependent on different pulse sequences. Motion artifacts cause slices to lose their relationship and cannot be regarded as a complete regular volume. In case of true 3D gradient echo sequences to capture a whole volume, any motion during acquisition may make the image not viable for registration. To overcome the motion artifact's effect, clinical setting of registration is designed to achieve fast and short acquisitions covering the whole volume to be imaged and frames with motion artifacts are excluded.

\subsection{Generalized Transformation Theory}

This approach performs alignment on the basis of some invariant landmark points on boundary of the target object (shape) [6] According to shape theory, if by transformation in a controlled environment the landmark points of one image can be mapped on the corresponding points of the other image then the two objects are of same shape. In case of inexact matching, approximation can be done by considering closest match. Geometrically invariant points are selected by considering points of high curvature on the boundary of the ROI [7].

A geometrical figure $\mathrm{X}$ in $\mathrm{R}^{\mathrm{K}}$ space consisting of $\mathrm{N}$ control points can be represented by $\mathrm{X}: \mathrm{N} \times \mathrm{K}$ matrix. Now from the concept of shape, two figures $X$ and $X^{\prime}$ have the same form if they are related by the following rigid body transformation equation [6].

$\mathrm{X}^{\prime}=\beta \mathrm{X} \Gamma+\mathrm{I}_{\mathrm{N}} v^{\mathrm{T}}$

$\begin{array}{ll}\text { where } & \\ \Gamma & : \mathrm{K} \times \mathrm{K} \text { is a rotation matrix and }|\Gamma|=1, \\ \mathrm{I}_{\mathrm{N}} & : \mathrm{N} \times 1 \text { of one, } \\ \nu & : \mathrm{K} \times 1 \text { is a translation vector, and } \\ \beta & : \text { isotropic scaling factor and }>=0\end{array}$

It is possible to formulate an approximate co-ordinate transformation for mapping between two sets of landmarks in a least square sense using Taylor series expansion. For two sets of landmark points $\left(\mathrm{x}_{\mathrm{m}}, \mathrm{y}_{\mathrm{m}}\right)$ and $\left(\mathrm{x}_{\mathrm{m}}{ }^{\prime}, \mathrm{y}_{\mathrm{m}}{ }^{\prime}\right), \mathrm{m}=1,2, \ldots, n$, one set can be expressed in terms of other as follows:

$x^{\prime}=q_{0}+q_{1} x+q_{2} y+q_{3} x^{2}+q_{4} x y+q_{5} y^{2}+\ldots$

$y^{\prime}=r_{0}+r_{1} x+r_{2} y+r_{3} x^{2}+r_{4} x y+r_{5} y^{2}+\ldots$

In terms of matrix, different components of x' can be expressed as

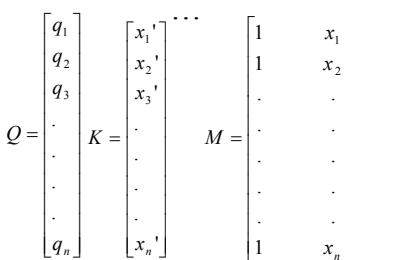

where solution of $\mathrm{Q}$ is given by $\mathrm{Q}=\left(\mathrm{M}^{\mathrm{T}} \mathrm{M}\right)^{-1} \mathrm{M}^{\mathrm{T}} \mathrm{K}$

Similarly, solution of $\mathrm{R}$ can be expressed as $\mathrm{R}=\left(\mathrm{M}^{\mathrm{T}} \mathrm{M}\right)^{-1} \mathrm{M}^{\mathrm{T}} \mathrm{L}$

The linear components of the equations 2 and 3 are related to the affine part and the higher order terms $\mathrm{q}_{3}, \mathrm{q}_{4}, \mathrm{q}_{5}, \mathrm{r}_{3}, \mathrm{r}_{4}, \mathrm{r}_{5}$ are related to the non-affine (non-linear) part of the transformation equations. For closed match of the landmark points, the least square solution for the coefficient matrix $\mathrm{Q}$ and $\mathrm{R}$ of the transformation are searched by maximizing the expression $\|\mathrm{MQ}-\mathrm{K}\|^{2}=0$.

So, we can say that affine and projective transformations are two special cases of generalized shape theory. In affine transformation, one needs at least 3 landmark points as it has 3 degrees of freedom (DOF), whereas projective transformation needs at least 4 control points.

\subsection{RBF neural network for implementation of image registration}

RBF network [8] is a multi dimensional interpolation model used to approximate an arbitrary function by means of a linear combination of basis functions. RBF networks belong to the class of kernel function networks where the inputs to the model are passed through kernel functions, which limit the response of the network to a local region in the input space for each kernel or basis function. The output from each basis function is weighted, summed and offset by some amount to provide the output of the network. The linear time-invariant transfer functions are comparatively less complex, more stable and robust than the nonlinear models. With all these advantages RBF networks provide a method to train a nonlinear model in terms of linear combination of basis functions. In our experiment with RBF, we have found that with a tolerance limit of $10^{-4}$, model generates and adds neurons to the hidden layer proportional to the number of inputs and trains up the self organizing structure much faster than an MLP model.

\section{DEMPSTER SHAFER THEORY OF EVIDENCE FUSION}

This model generalizes the Bayesian inference method. Analogous to the Bayesian method, the DS technique updates a prior mass density function to obtain a posterior evidence interval. The evidential interval quantifies the measure of belief of a proposition and its plausibility. Mass density functions provide the analogy to Bayesian probability.

It starts by assuming mutually exhaustive sets of propositions $\theta$, also called a Frame of Discernment (FOD) and the power set $\left(2^{\theta}\right)$. The elements of $2^{\theta}$ are the class of general propositions in the domain. DS approach assigns evidence mass ' $\mathrm{m}$ ' (basic probability assignment) on the subsets $A$ of the power set $2^{\theta}$. The subset $A$ can be singleton (or single proposition) such as $\left\{A_{i}\right\}$, or a composed proposition such as $\left\{\mathrm{A}_{\mathrm{i}} \mathrm{A}_{\mathrm{j}}\right\}=\mathrm{A}_{\mathrm{ij}}$. The evidence mass 
' $\mathrm{m}$ ' allows the set of symbolic classes of $2^{\theta}$ to be mapped into the numerical values of the interval $\left[\begin{array}{ll}0 & 1\end{array}\right]$ :

$$
\begin{aligned}
\mathrm{m}: 2^{\theta} & \rightarrow[0,1] \\
\mathrm{A} & \rightarrow \mathrm{m}(\mathrm{A})
\end{aligned}
$$

This mass function satisfies the following properties:

$$
\begin{aligned}
\mathrm{m}(\phi) & =0 \quad \text { where } \phi \text { is a null set } \\
\sum_{A \subset \theta} m(A) & =1
\end{aligned}
$$

The value $m(A)$ represents the degree of evidential support with which a specific element of $\theta$ belongs to the set $\mathrm{A}$.

A salient characterization of the DS theory is its powerful combination operator to create a pool of evidences coming in from various sources into a single belief figure for each hypothesis. In case of two bpas $\mathrm{m}_{1}$ and $\mathrm{m}_{2}$ associated with an FOD $\theta$, a new distribution of bpa $\mathrm{m}_{1,2}$ on $\theta$ is defined as

$m_{1,2}(S)=\sum m_{1}(A) \circ m_{2}(B)$

$$
\mathrm{s}=\mathrm{A} \cap \mathrm{B}
$$

A belief function assigns to each subset of $\theta$ a measure of our total belief in the proposition represented by the subset. A function m: $2^{\theta} \rightarrow[0,1]$ is called a belief function if it satisfies $\operatorname{Bel}(\phi)=0$. $\operatorname{Bel}(\theta)=1$, for any collection $A_{1}, A_{2}, \ldots A_{n}$ of subsets of $\theta$. Corresponding to each belief function there is one and only one basic probability assignment. Thus,

$$
\operatorname{Bel}(A)=\sum_{B \subseteq A} m(B), \forall A \subseteq \theta
$$

and $\mathrm{Bel}(\operatorname{not} \mathrm{A})$ is the Bel of the complement of $\mathrm{A}$. Bel(A) and $\operatorname{Bel}($ not $A)$ form only a part of all the subsets of $\theta$. Hence,

$\operatorname{Bel}(\mathrm{A})+\operatorname{Bel}(\operatorname{not} \mathrm{A}) \leq=1.0$

i.e. one can assign belief neither to $A$ nor to its negation but in case of classical probability model $\mathrm{P}(\mathrm{A})+\mathrm{P}(\operatorname{not} \mathrm{A})=1.0$

Belief interval characterizes the unassigned belief and hence the uncertainty associated with the hypothesis. The belief interval of a set $\theta$ is defined as:

$\mathrm{I}=[\operatorname{Bel}(\mathrm{A}), 1-\operatorname{Bel}(\operatorname{not} \mathrm{A})]$

with the properties as follows:
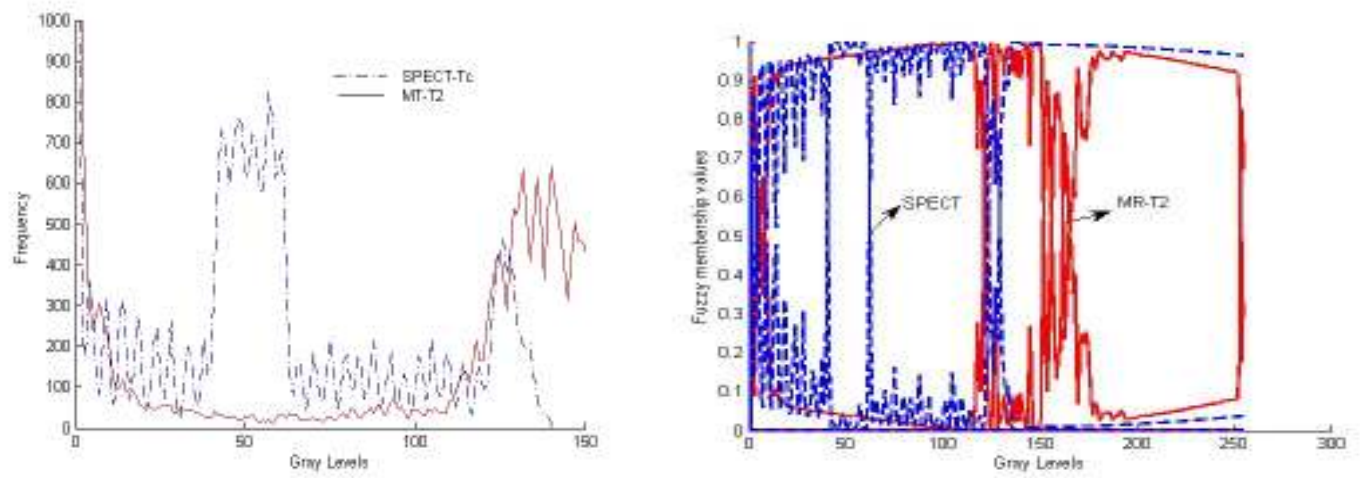

Figure 1: Gray level histograms of images and gray level vs. membership value plot max-length $[\mathrm{I}]=1.0$, and min-length $[\mathrm{I}]=0.0$

Maximum length is attained when one has belief neither in A nor in its negation, whereas minimum length is obtained where there is full belief to A or its negation. But if one has equal belief to A and to its negation then also the interval is of zero length and it is not possible to take any decision.

Plausibility $\mathrm{Pl}(\mathrm{A})$ is defined as the measure of belief that the true hypothesis is not contained in the complementary set of A and is written as

$$
P l(A)=\sum_{A \cap B \neq \phi} m(B) \forall A \subset \theta
$$

A new parameter $\mathrm{K}$ emerges when the assignment of evidence is made to conflicting propositions. $\mathrm{K}$ is interpreted as a measure of conflict between the sources and is directly taken into account in the combination as a normalization factor. To evaluate the quality of the combination, $\mathrm{K}$ value is taken into consideration.

Let $\mathrm{m}_{1}$ and $\mathrm{m}_{2}$ are two bpas over same FOD, and

$\mathrm{m}_{1}(\mathrm{~A}) \cdot \mathrm{m}_{2}(\mathrm{~B})<1$

$A \cap B=\phi$

Then the combined $\boldsymbol{b p a}$, is

$\mathrm{M}(\mathrm{C})=\left(\mathrm{m}_{1}+\mathrm{m}_{2}\right) \mathrm{C}$

$$
M(C)=\left(m_{1}+m_{2}\right) C=\frac{\sum_{A \cap B=C} m_{1}(A) \cdot m_{2}(B)}{1-\sum_{A \cap B=\phi} m_{1}(A) \cdot m_{2}(B)}
$$

and $\mathrm{M}(\mathrm{C})=0$ for $\mathrm{C}=\phi$

The conflict or normalization factor ' $\mathrm{K}$ ' appears for the assignment of evidence to conflicting propositions. If $\mathrm{S}_{1}$ assigns evidence $\mathrm{m}_{1}(\mathrm{~A})$ to proposition $A$ and sensor $S_{2}$ assign evidence $\mathrm{m}_{1}(\mathrm{~B})$ to a conflicting proposition $\mathrm{B}$ then

$$
K=\sum_{A \cap B=\phi} m_{1}(A) \cdot m_{2}(B)<1
$$

DS rule is consistent with the law of probability and the combination also results in a Bayesian mass function. Thus probability appears as a limit to DS, in the case where no ambiguity or imprecision exists [9]. 


\subsection{Fusion of multimodal images}

Fusion is an important step in the process of image registration. It gives a more informative combined image of two or more images. Medical image registration and fusion process increases the reliability of clinical decision-making mechanism [6]. In most of the cases on medical image, fusion provides anatomical localization of functional parameters. In case of functional to functional image fusion, combined image provides complimentary functional information of the source images. In all these situations, we have to deal with multiple information from different image modalities. Image processing under this circumstance needs data fusion techniques based on exploiting redundant and complementary information from different sources. We propose here an active fusion concept for segmentation of the combined image according to the intensity pattern of the corresponding pixels of the registered images.

The fusion is achieved by assigning the pair of pixels belonging to the corresponding coordinate points of both the images which represents the same physical coordinate point into one class $\mathrm{C}_{\mathrm{i}}$ of class set $=\left\{C_{i}\right\}_{i=1 . . N}$. This classification is performed by combining the bpa measures of the pixel pair of two images. The numerical measures of bpa correspond to the grey levels of the physical point $\mathrm{p}$ in their respective images and are assigned by fuzzy c-means. The point $p$ may be of two different classes $\left(C_{i}\right.$ and $\mathrm{C}_{\mathrm{j}}$ ) in two images with no prior connections. The combined FOD is obtained by the Cartesian product of the focal elements of two FODs. Each class $\left\{C_{k}^{12}\right\}$ of the resulting FOD is formed by a logical AND operation of corresponding classes of the component images. Then the combined $\boldsymbol{b p} \boldsymbol{a}$ is obtained by using the DS accumulation concept as stated in the equation 11. Since the combined mass value is in normalized form, the sum of mass values of all the classes of the resultant image is also 1 [9].

\subsection{FCM Clustering and Determination of Combined Mass Values}

FCM clustering algorithm $[10,11]$ provides unsupervised adaptive iterative technique for classification of image gray levels. FCM identifies cluster classes by iteratively modifying each class with respect to its distance between each measure of gray values and its class center. This is continued until any new gray level is assigned to any cluster class of FOD.

For medical images the grouping may be done on the basis of white matter (WM), grey matter (GM), cerebrospinal fluid (CSF) and ventricle, by assigning numerical values to each gray level $\mathrm{x}$ of $\theta$. Gray levels are grouped to different classes of FOD on the basis of the distribution of intensities over the histogram (Figure 1). FCM creates ' $n$ ' clusters $C_{i}$ of gray values and assigns ' $n$ ' membership values to each gray level (Figure 2). To find the bpa's of combined image a sum of product (equation 6) of membership values of $\mathrm{C}_{\mathrm{i}}$ is done by forming a the conflict matrix as stated in DS model. This gives $n \times m$ membership values of the resultant gray level of the fussed image and can be defuzzified by taking the largest membership value. The corresponding class is then considered as the cluster index of the fussed pixel. This approach produces a fused image with ' $\mathrm{n} \times \mathrm{m}$ ' clusters from two images of $\mathrm{n}$ and $\mathrm{m}$ clusters. The conflict resolution strategy of DS theory produces a normalized set of membership values for each gray level and maps each pixel pair to its corresponding cluster in the fused image. In this adaptive approach each class is defined in terms of gray level values and represented by FCM membership values distributed among the clusters.

\section{METHODOLOGY AND MATERIALS}

Anatomical to functional brain image registration and fusion have been implemented using generalized spatial transformation of objects of target images with respect to a set of invariant control point pair and Fuzzy-DS evidence accumulation method respectively. The proposed methodology is discussed here in brief.

\subsection{Construction of RBF neural model}

Here the RBF neural model is used as functional approximation to estimate the spatial transformation of unregistered image. This requires the determination and tuning of its architectural parameters according to the expected outcome from the network. Tuning of different unknown parameters in a particular RBF network includes determination of:

1. The number of hidden units,

2. Centers and widths of each basis function and

3. Output layer weights and basis values.

This type of radial neuron acts as a detector that produces one whenever the input vector is identical to its weight vector $w$. Thus to obtain the desired output, we have set the initial values of hidden layer weights equal to the desired values. The bias ' $b$ ' allows the sensitivity of the neuron to be adjusted and is set to $b\{1\}=\frac{\sqrt{-\operatorname{los}(.5)}}{c}$, where ' $c$ ' is a given constant. After each iteration, the actual outputs are compared against the calculated network outcome and the weight matrix is adjusted according to the learning rate, the difference and the activation function of the hidden layer. The training process continues till the convergence is achieved or the maximum iteration limits exceeds.

The network is trained using "supervised growing cells structure" learning algorithm [12]. The approach proceeds as follows:

1. Initiate a small RBF network

2. Train the network

3. Determine where a new RBF unit should be inserted from the errors in the training set.

4. Repeat until errors are small enough.

Insertion of new neuron takes place in regions between the spaces corresponding to the maximum error and it's nearest topological neighbours [12]. The resampling of mapped points was done by bicubic interpolation, as it produces smoother surfaces than the corresponding surfaces obtained by bilinear interpolation or nearest-neighbour interpolation. The bicubic interpolation was composed of piecewise cubic polynomials defined on subinterval $(-2-1),(-1,0),(0,1)$, and $(12)$. Outside this interval the interpolation kernel is zero. With this configuration the bicubic resampling was accomplished with a kernel of the form [13]:

$h(x)= \begin{cases}(a+2)|x|^{3}-(a+3)|x|^{2}+1 & 0 \leq|x|<1 \\ (a)|x|^{3}-5 a|x|^{2}+8 a|x|-4 a & 1 \leq|x|<2 \\ 0 & 2 \leq|x|\end{cases}$ 
To normalize the coordinate systems of reference and target image space, it is necessary to scale them to a common frame of reference. This makes the approximation of mapping function unbiased and balances the different influences of their origins. For this purpose we have scaled the input and output space by mapping them within a specific range and after the spatial transformation with the normalized input, the normalized network outputs were transformed back to their original co-ordinate system. Alternatively, normalized standard deviation or Principal Component Analysis (PCA) approache can also be used, but we have found that a function approximation on the basis of control point is better suited with min-max normalization.

We verified the used neural net with separately selected set of random points and continued the process with different adjusted sets of target parameters till the outcome is within the acceptable limit.

\section{EXPERIMENTS AND RESULTS}

\subsection{Registration of MR-T2 with functional brain images}

The size of the test PET images is $20 \%$ less and at an angle $5^{\circ}$ than that of the reference MR-T2. The size of SPECT image is $18 \%$ less than that of the size of base MR-T2 image and its angular shift is only $4^{\circ}$ from the base. Since PET/SPECT images lack morphological information, we have segmented the MR-T2 and PET/SPECT images to identify the corresponding anatomical locations (Figure 2) and selected three sets of control point pairs from 15 corresponding locations of both the images. Input points are then fine tuned on the basis of normalized cross-correlation of the neighbours of input and reference points. The training and validation of the neural network was done on the basis of these points (Figure 5). To verify the correctness of the registration we interchanged the role of input and reference images and performed the same excurse with the same set of control points. Then the trained RBF model was used to register the PET/SPECT and MR-T2 images.

\subsection{Fusion of MR with PET and SPECT brain images}

After alignment, we fused the images using DS evidence accumulation concept to get the final output from the registered images. The original images to be segmented and fused are shown in Figures $3 \& 4$. The resultant normalized images are of same size $(256 \times 256)$ and the image pixels are adaptively classified into three clusters, namely $\mathrm{C}_{1}, \mathrm{C}_{2}$ and $\mathrm{C}_{3}$.

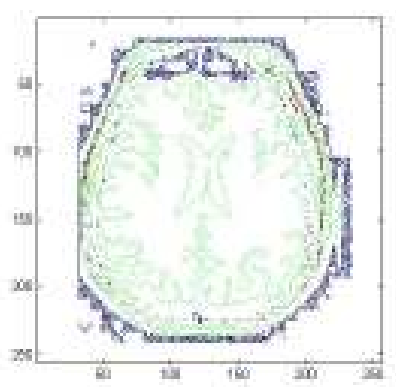

Figure 2: Segmented PET and MR T2 image used for identification of invariant control point pairs.

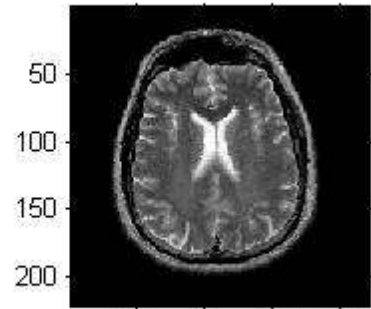

$50 \quad 100 \quad 150 \quad 200$

(a) Reference MR-T2

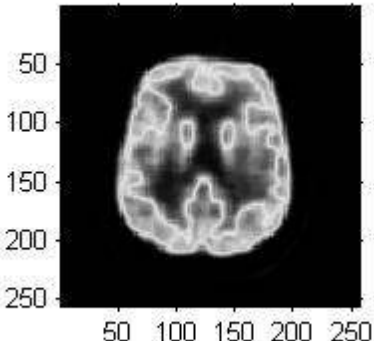

(b)Target PET

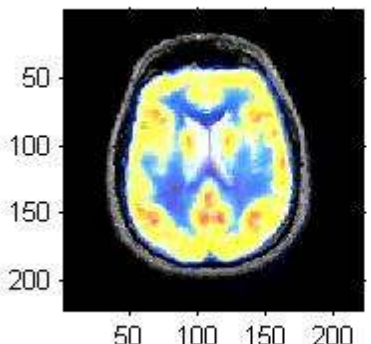

(c) Registered MR-T2 and PET images

Figure 3: Registration of MR (a) \& PET (b) images resulting in fused registered image (c) 
Here the FOD $\theta=\left\{C_{1}, C_{2}, C_{3}\right\}$ and the power set $2^{\theta}$ will be $\left\{\theta, C_{1}\right.$, $\mathrm{C}_{2}, \mathrm{C}_{3}, \mathrm{C}_{1} \cup \mathrm{C}_{2}, \mathrm{C}_{1} \cup \mathrm{C}_{3}, \mathrm{C}_{2} \cup \mathrm{C}_{3}, \mathrm{C}_{1} \cup \mathrm{C}_{2} \cup \mathrm{C}_{3}$ \}. The mass value ' $\mathrm{m}$ ' is assigned according to the membership values of the gray level to each individual class of FCM clusters.

$m_{1}: 2 \theta_{1} \rightarrow[0,1], m_{2}: 2 \theta_{2} \rightarrow[0,1]$

$\theta_{1}=\left\{C_{1}^{1}, C_{2}^{1}, C_{3}^{1}\right\}, \theta_{2}=\left\{C_{1}^{2}, C_{2}^{2}, C_{3}^{2}\right\}$

To explain the method we have considered an example point $\mathrm{p}$. The mass value of the given point $p$ of two images $I_{1}$ and $I_{2}$ are given in Table 1. Using this 'mass' distribution of the point $p$ and equation 6 , the combined mass distribution can be calculated (Table 2).

Table 1: Fuzzy mass values of the pixel $\boldsymbol{p}$ for three classes.

\begin{tabular}{|l|l|l|l|l|}
\hline Image & & $\mathbf{C}_{\mathbf{1}}$ & $\mathbf{C}_{\mathbf{2}}$ & $\mathbf{C}_{\mathbf{3}}$ \\
\hline MR-T2 & $\mathrm{m}_{1}$ & 0 & .8988 & .1012 \\
\hline PET & $\mathrm{m}_{2}$ & 0.0063 & $\phi$ & .9937 \\
\hline
\end{tabular}

Table 2 Conflict Matrix of the mass values of the point $\boldsymbol{p}$

\begin{tabular}{|l|l|l|l|l|}
\cline { 3 - 5 } \multicolumn{2}{l}{} & \multicolumn{3}{l|}{$\mathbf{2}^{\text {nd }}$ image } \\
\cline { 2 - 5 } \multicolumn{2}{l}{} & $C_{1}^{2}$ & $C_{2}^{2}$ & $C_{3}^{2}$ \\
\hline $\mathbf{1}^{\text {st }}$ image & .0063 & 0.0 & .9937 \\
\hline$C_{1}^{1}$ & .0 & 0 & 0 & .0 \\
\hline$C_{2}^{1}$ & .8988 & .0056 & 0 & .8931 \\
\hline$C_{3}^{1}$ & .1012 & .0006 & 0 & .1000 \\
\hline
\end{tabular}

Table 3 Membership values of $\mathrm{n} \times \mathrm{m}$ clusters

\begin{tabular}{|l|l|l|}
\hline $\begin{array}{l}\text { Fused } \\
\text { Classes }\end{array}$ & $\begin{array}{l}\text { Symbolic } \\
\text { Representation }\end{array}$ & Bpas \\
\hline$C_{1}^{1} \cap C_{1}^{2}$ & $C_{1}$ & 0 \\
\hline$C_{1}^{1} \cap C_{2}^{2}$ & $C_{1,2}$ & 0 \\
\hline$C_{1}^{1} \cap C_{3}^{2}$ & $C_{1,3}$ & 0 \\
\hline$C_{2}^{1} \cap C_{1}^{2}$ & $C_{2,1}$ & .0056 \\
\hline$C_{2}^{1} \cap C_{2}^{2}$ & $C_{2}$ & 0 \\
\hline$C_{2}^{1} \cap C_{3}^{2}$ & $C_{2,3}$ & .8931 \\
\hline$C_{3}^{1} \cap C_{1}^{2}$ & $C_{3,1}$ & .0006 \\
\hline$C_{3}^{1} \cap C_{2}^{2}$ & $C_{3,2}$ & 0 \\
\hline$C_{3}^{1} \cap C_{3}^{2}$ & $C_{3}$ & .1000 \\
\hline
\end{tabular}

The final distribution of masses $\mathrm{m}_{1,2}=\mathrm{m}_{1} \oplus \mathrm{m}_{2}$ are calculated using the values of Table 2 and shown in the Table 3 .

Since FCM creates clusters adaptively on the basis of intensity distribution only, classes $C_{i, j}$ and $C_{j, i}$ are not same, there will be nine clusters and the pixel of our interest will belong to the class $C_{2,3}$.

Figure 3 shows common regions of both the images with PET image showing functionally hypo-active regions. Four classes (gray matter, white matter, cerebrospinal fluid and tumor) are considered to classify the images and FCM algorithm is used to classify the individual images into desired clusters. With the help of DS approach we calculated the mass value of each combined pixel. From the four classes using DS conflict resolution approach we got sixteen classes of the combined image. Each of the sixteen classes is assigned different color codes to get final fused image (Figure 3(c)).

In the Figure 4 the MR image demonstrates the target areas with mixed signal intensity and the SPECT-Tl image shows the corresponding areas of hypometabolism with low Thallium uptake. The fused image in the Fig. 4(c) shows different functionally active regions of SPECT image over the MR image. Here also, both the images are classified into four classes using FCM before fusing those using DS conflict resolution strategy.

\subsection{Synergistic study of the fused image}

From the experimental outcome of MR-PET and MR-SPECT registration (Figures $3 \& 4$ ) we have found that the anatomical localization of functional brain activities are quite satisfactory. The MR-PET image (Figure 3) shows hypometabolism in anterior temporal and posterior temporal regions. The abnormal cerebral metabolism as shown in PET image is distinctly visible over MRI.

It is clear from the MRI-guided SPECT measurements that there is considerable amount of decrease of $\mathrm{CBF}$ or perfusion in the parietal lobes area (Figure 4). The infected brain areas (intra parietal brain sulci and parietal lobules) are also distinctly identifiable from the MRI. The ROIs with glucose hypometabolism are clearly outlined in the registered image (Figure 3(c)). The clinical assessment of the enlarged right ventricular portion of MR image (Figure 3(a)) is also possible for diagnosis and therapeutic planning

\subsection{Validation of Registration and Fusion methodologies}

Validation of registration is required to accept the result clinically. Accuracy is measured in terms of visual inspection of result with respect to a ground truth. We have created one ground-truth GT1 using MATLAB image registration toolbox and compared it with the MR-PET/SPEC registration outcomes. The comparison was done by evaluating the differences between computed and reference transformation at selected and well-distributed points near the brain surface to obtain an estimate of the upper bound of the registration error within the brain volume of interest.

Our registration algorithm is capable of consistently aligning both low and high resolution MR, PET and SPECT images with subvoxel accuracy. The average error for PET to MR, SPECT to MR is half the largest voxel size and in only $5 \%$ of the cases it was different with the control point based registration solution. Linear regression between the outcome of proposed transformation equation and the target produces a correlation coefficient R very close to unity (Figure 6).

The proposed fusion provides us a way to eliminate the conflicting conditions and fails only if the bpa's have contradictory mass values in two different classes. FCM over intensity values is a robust clustering mechanism and produces 
stable and consistent outcomes for different sets of input parameters.

The performance of the proposed approach is quite satisfactory. Visual inspection of the fused-registered image of Figures 3(c) and $4(\mathrm{c})$ show that the quality of the registered image is within the acceptable limit. Moreover, similarity measure of the transformed input points with the original values shows a close similarity (Figure 5(b)).

\section{CONCLUSION}

Here we have suggested a computational platform for $\mathrm{AD}$ brain image study on the basis of registered and fused anatomicalfunctional brain images. The approach is a neuro-fuzzy method where we have used RBF neural network model to implement the registration of MR-PET/SPECT brain images and then used FCM clustering method to classify images into the target classes before combining candidate images using DS evidence accumulation method. This method of fusion provides us a mathematical framework for measuring uncertainty, imprecision and conflicting situations. Moreover, this is also robust in the sense that only inputs from different multimodal images are needed for the classification. The neural network provides a nonlinear, flexible and robust multimodal image registration platform. Finally, the high quality outcomes of the resultant fused images have shown this as a promising tool for synergistic study of AD brain images.

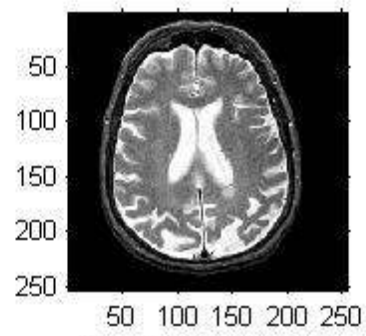

(a) Reference MR-T2

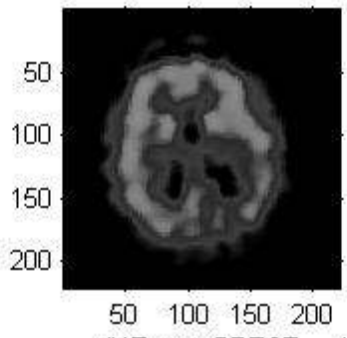

(b) Target SPECT

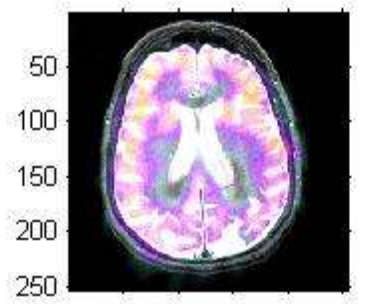

50100150200250

(c) Registered MR-T2 and SPECT images

Figure 4: Registration of MR (a) \& SPECT (b) images resulting in fused registered image (c)

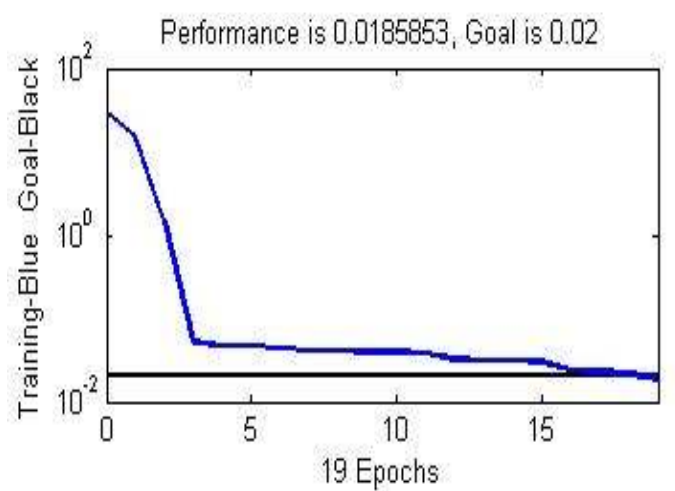

(a)

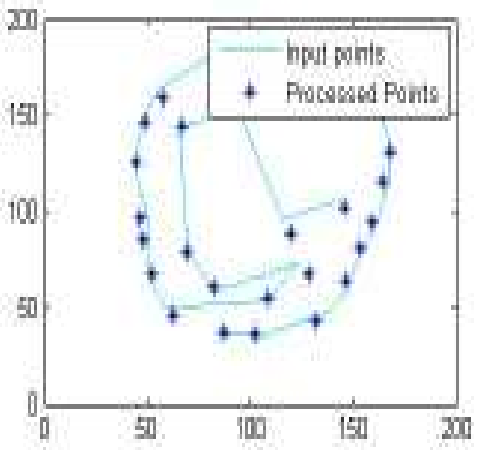

(b)

Figure 5:(a) Performance plot of the RBF network (b) Comparison of original and computed points of MR-SPECT image registration
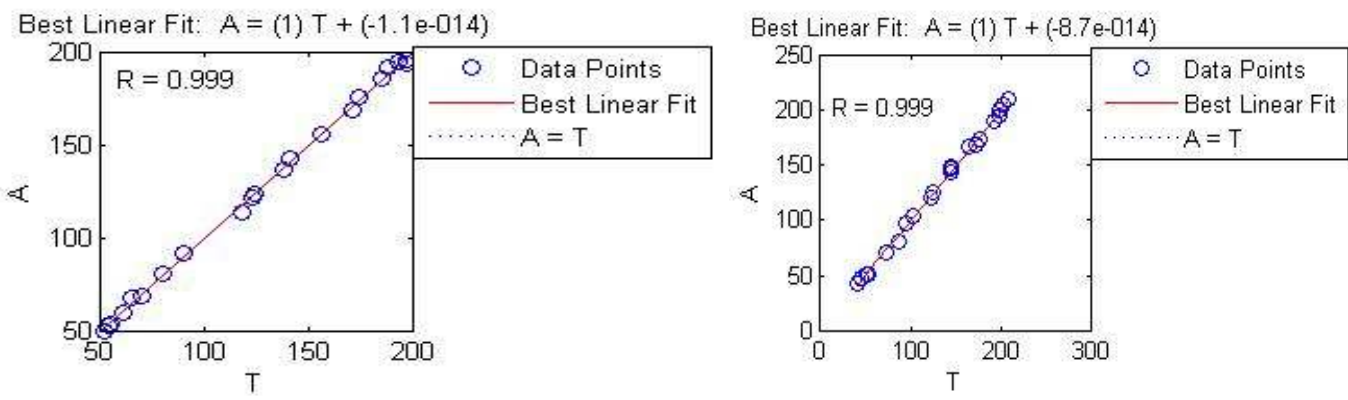

Figure 6: Validation of registration process using linear regression. 


\section{REFERENCES}

1. H.Henrik Ehrsson,Johann P. Kuhtz-Buschbeck, and Hans Forssberg, Brain Regions Controlling Nonsynergistic versus Synergistic Movement of the Digits: a Functional Magnetic Resonance Imaging Study, The Jr. of Neuroscience, June 15, 2002, Vol 22(12), pp 5074-5080.

2. Pascal Kurosinski. Mathias Guggisberg, and Jurgen Gotz, Alzheimer's and Parkinson's disease - overlapping or synergistic pathologies?, Trends in Molecular Medicine, Vol 8(1), January 2002, pp 3-5.

3. Medha V. Wyawahare, Dr. Pradeep M. Patil, and Hemant K. Abhyankar, Image Registration Techniques: An overview, International Journal of Signal Processing, Image Processing and Pattern Recognition Vol. 2, No.3, September 2009.

4. G Shafer. A Mathematical Theory of Evidence. Princeton University Press, 1976.

5. C R Parikh, M J Pont, N B Jones, Application of DempsterShafer theory in condition monitoring systems: A Case study, Patter Recognition Letters, 2001,22(6-7), 777-785.

6. D Dutta Majumder, A Study on a Mathematical Theory of Shapes In Relation To Pattern Recognition and Computer Vision, Indian Journal of Theoretical Physics 43(1995) 1930 .
7. M Bhattacharya, D Dutta Majumder, Registration of CT and MR images of Alzheimer's patient: A shape theoretic approach. Pattern Recognition Letters, vol. 21, pp. 531-548, 2000 .

8. M J D Powell. Radial basis functions for multivariable interpolation: a review. In Algorithms for Approximation. 143-167. Clarendon Press, Oxford, 1987.

9. J Timothy Ross, Fuzzy Logic with Engineering Applications, McGraw-Hill International, 1997.

10. J C Bezdek, R J Hathaway, M J Sabin and W T Tucker, "Convergence theory for fuzzy C-means: counter examples and repairs", IEEE Trans. Syst. Man Cybern, 1987, 17873-7.

11. G Beni and X Liu X, A least biased fuzzy clustering method IEEE Trans. Pattern Anal. Machine Intell. 1994, 16 954-60.

12. B Fritzke, Supervised learning with growing cell structures. In J.D.Cowan, G.Tesauro and J.Alspector. Editor, Advances in Neural Information Processing Systems, Vol.6, 255-262, Morgan Kaufmann. Publishers Inc., San Mateo, CA, 1994.

13. R Keys, "Cubic Convolution Interpolation for Digital Image Processing", IEEE Transactions on Acoustics, Speech and Signal Processing, Vol.ASSP-29, No.6, December 1981, p. 1155 . 